\title{
Troponin $I$ is encoded in the haplolethal region of the Shaker gene complex of Drosophila
}

\author{
Julio A. Barbas, Joan Galceran, Imke Krah-Jentgens, ${ }^{1}$ José Luis de la Pompa, Inmaculada Canal, Olaf \\ Pongs, ${ }^{1}$ and Alberto Ferrús \\ Instituto Cajal, CSIC, Madrid 28002, Spain, ${ }^{1}$ Lehrstuhl für Biochemie, Ruhr-Universität Bochum, Germany
}

\begin{abstract}
We have analyzed one of the nine complementation groups that constitute the haplolethal (HL) region of the Shaker gene complex (ShC). Five mutations, including a dominant lethal, define this complementation group: HL I. Mutant phenotypes show abnormal embryogenesis with structural defects in the nervous system and aberrant degeneration of specific adult muscles in addition to altered action potentials. HL I encodes a family of proteins with extensive homology to invertebrate troponin I (TnI). Members of this family are brought about by two alternative and two mutually exclusive exons in conjunction with two differential polyadenylation sites. Transcription analysis indicates that some isoforms are adult specific and others are synthesized throughout development, except during early metamorphosis. Certain isoforms of Drosophila TnI are expressed in specific muscles. The specificity of mutant phenotypes suggests a functional role of particular TnI isoforms in the development and the mature activity of muscle and nervous systems.
\end{abstract}

[Key Words: Troponin I; Shaker gene complex; haplolethality; muscle]

Received June 4, 1990; revised version accepted October 25, 1990.

Shaker is a gene complex $(\mathrm{ShC})$ of Drosophila that includes the structural gene for a family of potassium channels (Pongs et al. 1988; Schwartz et al. 1988; Ferrús et al. 1990). It spans $350 \mathrm{~kb}$ and can be divided into three main regions: maternal effect $(\mathrm{ME})$, viable $(\mathrm{V})$, and haplolethal $(\mathrm{HL})$. It harbors a number of mutations distributed in 13 complementation groups with phenotypic similarities such as abnormal action potentials and neural development (Tanouye et al. 1981; Ferrús et al. 1990). The action potentials in the cervical giant interneuron (CGF) have been studied in representative alleles of four of these complementation groups and have been shown to be abnormal in duration or frequency (Tanouye and Ferrús 1985). One complementation group maps in the $\mathrm{V}$ region and corresponds to a 130-kb-long transcription unit encoding a family of A-type $\mathrm{K}^{+}$ channels (Iverson et al. 1988; Pongs et al. 1988; Schwartz et al. 1988). The other complementation groups that affect the frequency of action potentials map either to the ME or HL regions, outside the structural gene for $\mathrm{K}^{+}$channels.

The HL region is defined by the breakpoints of two chromosomal rearrangements associated with a Shaker phenotype: $T(X ; Y) W 32$ and $T(X ; Y) V 7$ (Tanouye et al. 1981). These breakpoints are separated by $170 \mathrm{~kb}$. Extensive genetic analysis of the $S h C$ indicates that the HL region is composed of nine lethal complementation groups (Ferrús et al. 1990). Their mutants exhibit genetic interactions in trans-heterozygotes with mutations in other regions of the complex, and some of them show abnormal action potentials and leg shaking, similar to the $\mathrm{V}$ mutations. One of the complementation groups in the $\mathrm{HL}$ region, $\mathrm{HL} \mathrm{I}$, is particularly interesting because it contains the expected dominant lethal mutations. In addition, HL I contains semidominant and recessive lethal mutations and viable mutations. The phenotypic analysis of some HL I mutants suggests that the biological functions encoded in HL I should be involved in neural and muscle pattern formation, as well as proper membrane repolarization after action potential firing. The developmental phenotypes of these mutations include aberrant fasciculation and projection pathways in the embryonic central nervous system (CNS) and musclespecific degeneration in the adult.

In this work we have investigated the transcription of the HL I DNA to understand the molecular bases of these phenotypic traits. We find that HL I encodes developmentally regulated isoforms of troponin I (TnI). This is a protein that forms multimers with troponins $\mathrm{C}$ and $\mathrm{T}(\mathrm{TnC}$ and $\mathrm{TnT})$ and tropomyosin $(\mathrm{Tm})$, which are known to regulate muscle contraction (Perry 1979). Although at present we have evidence of TnI expression only in muscle tissue, the HL I mutant phenotypes indicate that in addition to the role in muscle function, some TnI isoforms could be required also for early embryonic development as well as in neural function. 


\section{Results}

\section{Mutant phenotypes of HL I alleles}

The viable mutation heldup $(h d p)$ causes selective degeneration of adult thoracic muscles (Hotta and Benzer 1972; Deak et al. 1982). This mutation has been located between the $W 32$ and $V 7$ breakpoints /Homyk and Emerson 1988), corresponding to the HL region of ShC. The allele $h d p^{2}$ strongly alters the dorsolongitudinal muscles (DLMs) and dorsoventral muscles (DVMs) and slightly alters the tergal depressor of the trochanter (TDT) muscle (Fig. 1A,B). This phenotype becomes more extreme in trans-heterozygous $1(1) 1614 / h_{d p^{2}}$ flies (Fig. 1C). In this case most thoracic muscles, including TDT and other small tubular muscles, are absent or severely aberrant in their structure. However, to ascribe the muscle phenotype to the HL I transcription unit it was important to study other trans-heterozygous combinations, not including $h d p$ alleles. Female $1(1) 1614 /+\mathrm{mu}-$ tants exhibit a clear specificity for TDT. This muscle appears hyperplasic and poorly structured, whereas DLM and DVM are practically unaffected. This dominant trait is dosage dependent since female $1(1) 1614 /+$; $D p(1 ; 3) / C 153 /+$ mutants show normal muscles. However, the genotype 1(1)1614/1(1)9916; Dp(1;3)/C153/+ shows again the TDT-specific effect (Fig. 1D), suggesting that both lethal mutations are allelic with respect to the TDT muscle phenotype. In this genotype the $D p(1 ; 3) / C 153$ is needed to cover the dominant lethality of 1(1)9916 (see below). Other genotypes analyzed histologically confirm these observations and are listed in Materials and methods.

A further definition of the HL I complementation group was based on the complementation of viability between the lethal mutations 1/1)1614 and 1(1)8384. The dominant lethality of 1(1)9916 prevents most of the standard genetic tests of allelism; however, the semidominant lethal mutation 1(1)8384 allowed such tests. On the basis of the $0 \%$ viability of $1(1) 8384 / 1(1) 1614$, both mutations can be associated with the same complementation group as $h d p$ and 1(1)9916.

The dominant [1(1)9916] and semidominant [1(1)8384] lethal mutations of HL I have a very similar embryonic phenotype. The hypoderm appears to develop normally except in spots of the postgnathal-thoracic region, where only a thin layer of cuticle, but no hypoderm or muscles, is found. Abnormal embryonic development can be detected in 1/1)9916 as early as the blastoderm stage. The yolk is distributed irregularly in the cephalic region (Fig. 1E), and as development proceeds, the 21-hr

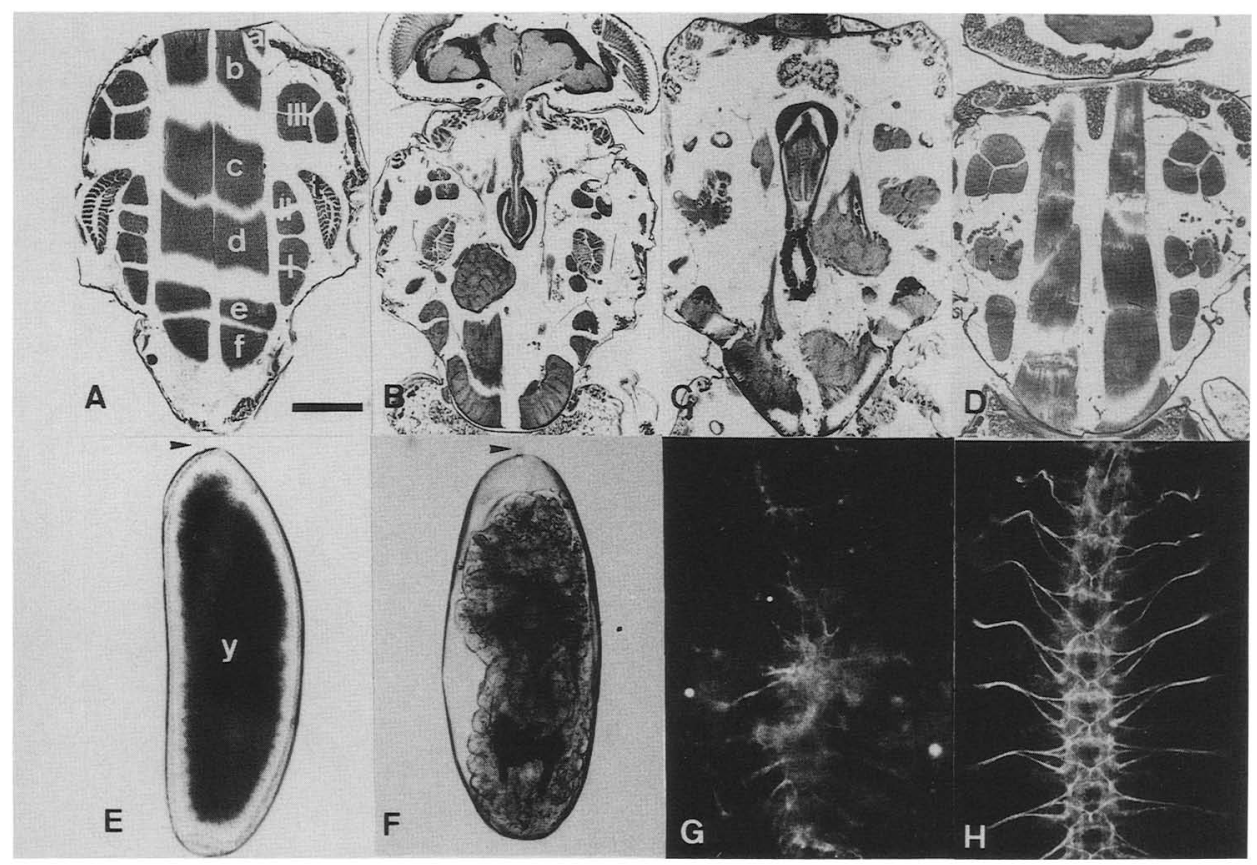

Figure 1. Phenotypes of HL I mutants. $(A)$ Horizontal section of a normal thorax showing the fibrillar muscles DLM with six fibers $(a-f)$, the DVM I (I) composed of two fibers, the DVM II (II) composed of two fibers, and the DVM III (III) with three fibers. Also, the tubular muscle TDT $(t)$ can be seen. $(B)$ Horizontal section of a $h d p^{2}$ male. Note the reduced fibrillar muscles and the slight effect in the TDT. $(C)$ Horizontal section of a $h d p^{2} / 1(1) 1614$ female. Note the stronger phenotype including the disappearance of TDT. $(D)$ Horizontal section of a 1(1)1614/1(1)9916, Dp (1;3)JC153/+ female. Note the rather selective and strong effect on TDT and the slight effect on DVM II (see text). (E) In vivo observation of $1(1) 9916$ embryogenesis (see Materials and methods). Blastoderm stage with irregular profile of the yolk $(\mathrm{y})$ material, especially in the cephalic region. The arrowhead points to the micropile. $(F)$ Final stage of 1(1) 9916 male embryo. Note the aberrant organization of the cephalothoracic tagmata; the abdominal ones are less affected. (G) Whole mount of a 1(1)9916 CNS stained with neural-specific mAb 22C10 (Fujita et al. 1982). Note the severe distortion of the metameric pattern and the almost complete lack of fasciculation among nerve fibers. $(H)$ Normal aspect of the CNS at a similar time of development as in $G$ for comparison. Anterior is to the top. Bar, $250 \mu \mathrm{m}$ in $A-D ; 180 \mu \mathrm{m}$ in $E$ and $F$, and $150 \mu \mathrm{m}$ in $G$ and $H$. 
embryo has a gross distortion of the anterior /cephalic and thoracic) half of the body. Interestingly, the abdominal segments are affected less severely (Fig. 1F). Wholemount preparations of the 15 -hr CNS stained with the neural-specific mAb 22C10 (Fujita et al. 1982) illustrate the abnormal structure of the nervous system (Fig. 1G). The CNS has almost no trace of its metameric pattern, and axons do not fasciculate and follow aberrant paths. Also, in isolated sensory neurons, supernumerary processes can be seen as compared to the normal type (not shown). The severity of this phenotype is diminished in a class of lethal embryos that we take as heterozygous 1(1)9916/ + females. The neural aberrations described should correspond to the lack of function(s) in the HL region since the $1(1) 9916$ phenotype is very similar to that of $D f(1) W 32^{D} / V 7^{P}$ (Ferrús et al. 1990).

In addition to these developmental effects, the HL I mutant 1(1)1614 shows physiological phenotypes in mature neurons. $1(1) 1614 /+$ females shake their appendages. A subsequent analysis of the CGF action potentials showed that they are fired in multiple spikes in contrast to the wild type, where they appear as single events (Fig. 2). This abnormality is very similar to that shown by other $\mathrm{V}, \mathrm{HL}$, and ME mutations. Also, a previous study of 1(1)1614 in gynandromorphs had shown that the ipsilateral side of male (mutant) territory was frequently associated with abnormal posture or movement of appendages (Ferrús et al. 1990).

\section{Physical mapping of HL I alleles}

In the process of studying the $S h C$, the entire DNA covered by $D p(1 ; 3) / C 153$, including the $W 32-V 7$ interval, has been cloned. The HL region is contained within 170 $\mathrm{kb}$ (Fig. 3). Systematic Southern analysis of mutant and wild-type DNAs, with probes spanning the whole HL region, were used to map the physical location of mutations at the HL I complementation group (not shown). The x-ray-induced 1(1)9916 mutation has an inversion with the proximal breakpoint at position 201-205 (Fig.
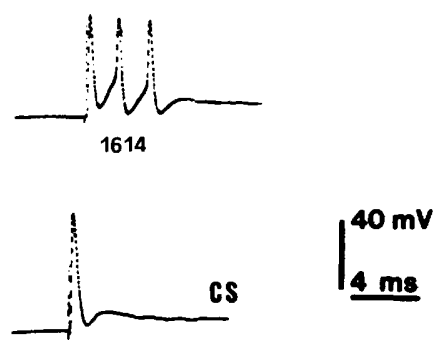

Figure 2. Action potentials from normal (CS) and HL I mutant [1(1)1614] adults. The mutant profile corresponds to a female 1(1)1614/B137D. $T(X ; Y) B 137$ breaks proximal to $T(X ; Y) V 7$ and distal to the proximal breakpoint of $D p(1 ; 3) / C 153$ (Ferrús et al. 1990). A similar abnormality in spiking frequency was found in other 1(1)1614 heterozygotes, as well as other ShC lethal mutations. The recordings were obtained from the CGF after brain stimulation following the procedure described in Tanouye and Ferrús (1985). (Modified from Ferrús et al. 1990.)
3). No further abnormalities were detected in Southern analysis. The distal breakpoint was determined by inspection of salivary gland chromosomes and is located in region 14A. Another x-ray-induced mutation, 1(1)8384, presents a $6-\mathrm{kb}$ insertion at position 209 (Fig. 3). The ethylmethane sulfonate (EMS)-induced alleles 1(1)1614 and $h d p^{3}$ did not show any alteration in Southern analysis.

\section{cDNA isolation and genomic structure of the HL I transcription unit}

Northern blot analysis using HL genomic probes revealed the existence of several transcription units within the region (not shown). One of them is found between map coordinates 200 and 215 (Fig. 3). This interval contains the detected alterations for 1(1)9916 and 1(1)8384. Screening of different libraries with genomic fragments within this interval rendered the cDNA clones E2, from 3- to 12-hr embryo; L9, from third instar larva; and A6, A16, from adult libraries, respectively. The four clones and the genomic fragments that hybridize to them have been sequenced. The resulting exon/intron organization is shown in Figure 3 . All of the flanking intron borders show the consensus $5^{\prime}$ - and $3^{\prime}$ splice sequences (Padgett et al. 1986). The four cDNAs start their sequence at the same nucleotide, which has been numbered 1 in Figure 4. No TATA-box like sequence is found upstream of this nucleotide. However, the putative enhancer sequence GGGCGG (positions -124 to -119 in Fig. 4) is typical of no TATA-box-containing genes in vertebrates (Kadonaga et al. 1986). All isolated cDNAs share exons 1, 2, 4, 5, 7, 8, and part of exon 10 (for details, see the legend to Fig. 4).

\section{Northern blot analysis and tissue expression}

Poly(A) ${ }^{+}$RNA, extracted from wild-type individuals at different developmental stages, was analyzed in Northern blots (Fig. 5). When probed with the cDNA E2 (Fig. 5a), the expression of HL I mRNAs can be detected from the embryonic stage onward. Two major bands within the range of $1.0-1.2 \mathrm{~kb}$ are observed throughout larval stages. At pupariation, these mRNAs disappear abruptly (lane PI). The expression resumes at a higher level in later pupal stages (lane PII) and persists during the adult phase. However, in addition to the embryolarval forms, the postmetamorphosis RNA showed larger RNAs ranging up to $1.4 \mathrm{~kb}$. In the adult stage, the $1.2-\mathrm{kb}$ mRNA is the predominant form. The same Northern blots used in this analysis were hybridized with $S h C$ and tubulin probes to monitor for equal amounts of RNA loaded in each lane (not shown).

Developmental Northern blots were also hybridized with specific probes for exons 3, 6a, 6b, and 9 /see Materials and methods). Exons 3 and 9 are present in an adult cDNA (A16). No expression of these exons is detected before metamorphosis [Fig. 5b (A,D)]. They hybridize only to adult and pupal mRNAs and specifically to the 1.2- and 1.4-kb bands. A similar pattern is observed with 

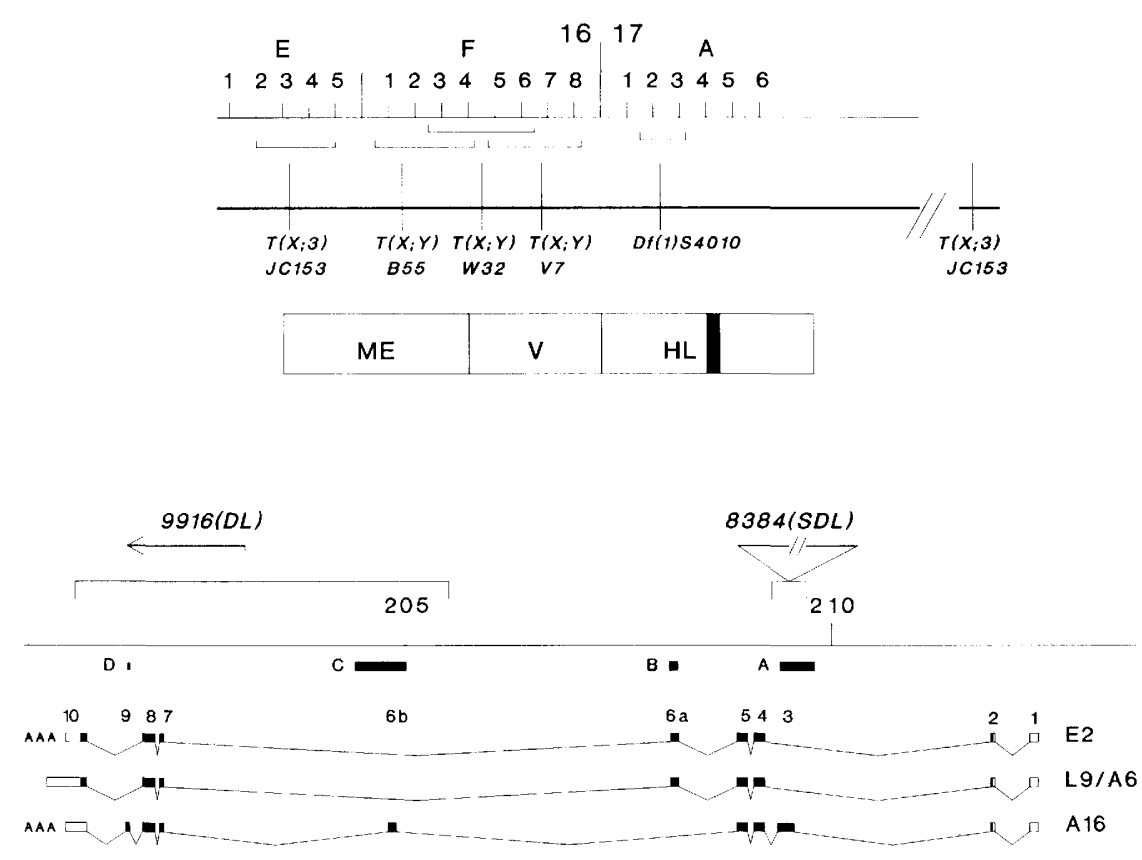

Figure 3. (Top) Cytogenetic map of the ShC. A schematic drawing of the salivary gland chromosome for this region is shown. The breakpoints of five chromosomal rearrangements used to localize $S h C$ cytogenetically are indicated by brackets (Tanouye et al. 1981). Centromere is to the right. The genetic organization of $S h C$ into ME, $\mathrm{V}$, and $\mathrm{HL}$ regions is indicated by open bars. The three regions span $350 \mathrm{~kb}$. (Bottom) Structures of four different cDNAs (E2, L9, A6, and A16) aligned with genomic Shaker region from which they derive. The coordinates on the genome follow those defined in Baumann et al. (1987) (calibration is in kb). The extension of the genomic fragments affected by inversion 9916 and insertion 8384 is shown. The map shows in detail a part of the HL region (vertical black rectangle). Small boxes indicate exons. They are open when corresponding to nontranslated regions. Exon numbers are in the direction of transcription. $(A-D)$ The extension of the genomic fragments used as exon-specific probes in Northern blots. exon 6b. It shows a major hybridization to 1.2- and 1.4$\mathrm{kb}$ mRNAs from pupal and adult stages, although a minor hybridization is also observed to the $1.0-\mathrm{kb}$ band [Fig. 5b (C)]. After long overexposure of the same blot, a weak signal could be detected in the mRNAs corresponding to the embryo-larval stages (not shown). Exon $6 \mathrm{a}$ is expressed throughout development (except in early pupal stages), but the 1.4-kb band is not detected at any stage [Fig. 5b (B)].

The heterogeneity of exon composition and the differential use of at least two polyadenylation sites is sufficient to account for the mRNA size distribution observed. The two RNAs found in embryo-larval stages $(1.0$ and $1.2 \mathrm{~kb})$ correspond in size to the two isolated cDNAs (E2 and L9), which differ only in their polyadenylation sites. The addition of exon 3 to these isoforms would generate two new mRNAs of 1.2 and $1.4 \mathrm{~kb}$, respectively. This complete set of four classes of mRNAs agrees with the pattern observed in adult and pupal stages. Exons $6 \mathrm{a}$ and $6 \mathrm{~b}$ have the same length and would have no contribution to the variable length of the different RNAs that come from this transcription unit. The presence of exon 9 would have a negligible effect on the length of the RNAs since it has only 43 nucleotides.

In situ expression of HL I RNA was ascertained using a riboprobe containing exons common to all isoforms known to date (Fig. 6). The signal can be detected in the mesoderm of a 12-hr embryo, as well as in all fibrillar and tubular muscles of larvae and adults. In contrast, an exon 3 riboprobe shows the presence of these isoforms only in the fibrillar muscles of the adult (DLM and DVM).

\section{HL I encodes isoforms of TnI}

The longest open reading frame contained in the cDNA sequences starts with the ATG codon at position 135 , within exon 2. Two other ATG codons are in-frame at positions 390 and 432 , but the one at position 135 is the first ATG codon that appears in the cDNA sequence and the only one with a consensual A residue 3 nucleotides upstream (Kozak 1984). For these reasons, this is the most probable initiation site of translation. cDNAs E2, L9, and A6 stop their coding sequences within exon 10. The deduced polypeptide (p6a10) has 208 amino acids and an estimated $M_{\mathrm{r}}$ of $24,479 \mathrm{~g} / \mathrm{mol}$. The most apparent feature of this deduced polypeptide is the high proportion of charged residues $(50 \%)$, in which basic residues account for $60 \%$ of them. cDNA A16 presents the addition of exons 3 and 9 within the coding sequence. Exon 3 incorporates a 61 -amino-acid-long extra domain near the amino terminus. The last nucleotide of exon 9, together with the first two of exon 10, creates an in-frame stop codon that leaves this last exon completely untranslated. In this way an alternative carboxyl terminus is generated. The new polypeptide (p36b9) has an estimated $M_{\mathrm{r}} 28,825 \mathrm{~g} / \mathrm{mol}$. The composition of the extra domain encoded in exon 3 differs significantly from the rest of the protein. It is very rich in alanine and proline residues (i.e., 30 and 12 of the 61 amino acids, respectively). Garnier plots of secondary structure show for both isoforms that $>90 \%$ of the residues are in the $\alpha$ helix conformation.

The deduced polypeptides show high homology with the reported amino acid sequence of TnI from crayfish (Kobayashi et al. 1989) (Fig. 7). When aligned, the sequence of TnI from crayfish and isoform p6a10 reported here have $65 \%$ identity. This value is in the range of that found among the different forms of vertebrate TnI available in the NBRF data bank (different muscle types from quail, chicken, and rabbit). However the homology is only $25-30 \%$ when compared with vertebrate TnI. Nev- 

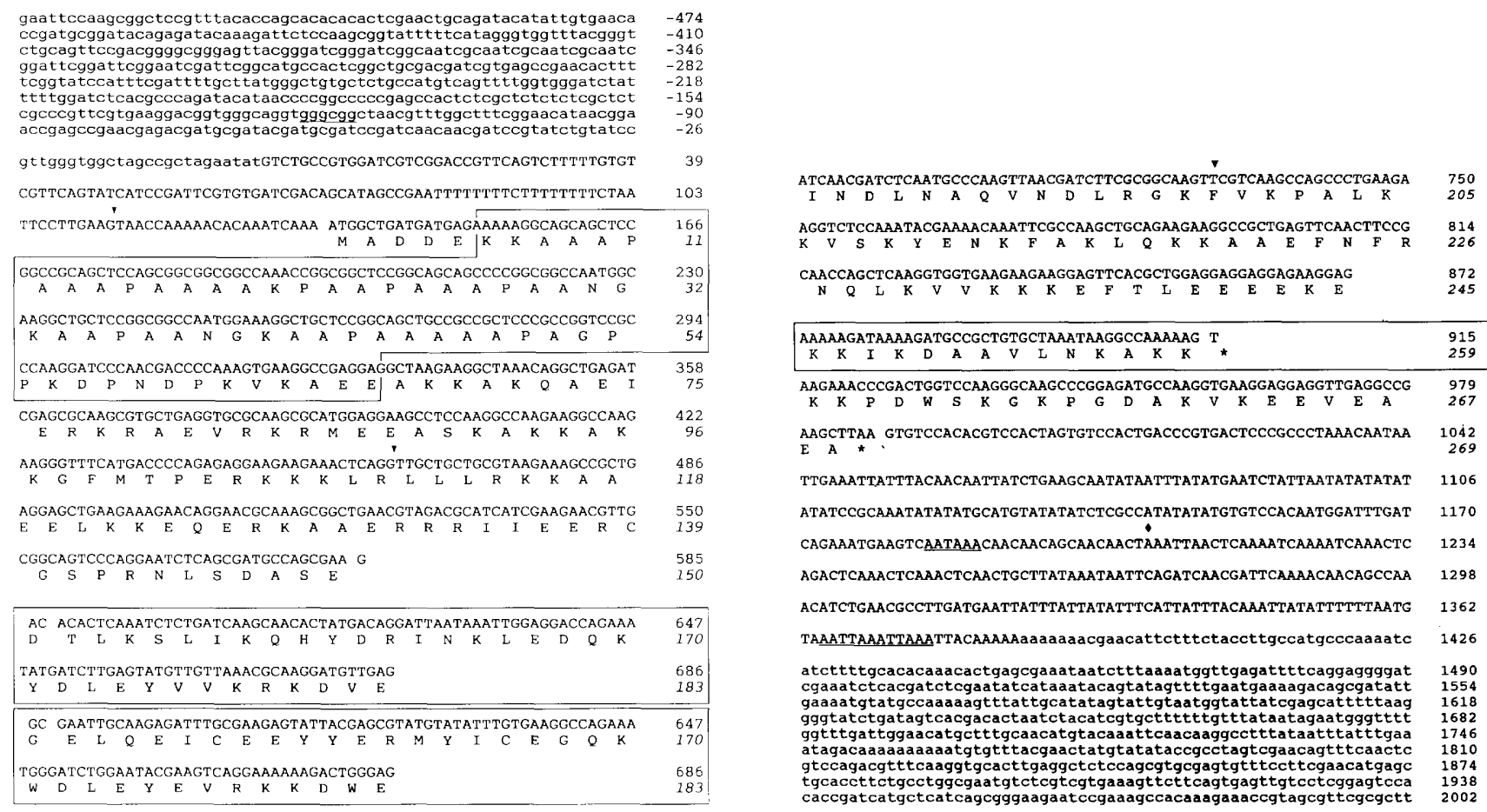

Figure 4. Partial nucleotide sequence of the HL I transcription unit. cDNA sequences are given in uppercase and genomic DNA is shown in lowercase letters. Position 1 corresponds to the first residue found in all of the cDNAs obtained. The upstream genomic sequences are shown in negative numbers. The arrowheads point to the limits between constitutive exons. Exons $3,6 \mathrm{a}, 6 \mathrm{~b}, \mathrm{and} 9$ (alternative exons) are boxed. Translation into amino acids is given from ATG codon at position 135. Note that numeration of nucleotides and amino acids considers the longest possible sequence. Exons $6 \mathrm{a}$ and $6 \mathrm{~b}$ are mutually exclusive in terms of nucleotides and amino acids. Amino acid sequence deduced from exon 9 is mutually exclusive with that of exon 10. No consensual TATA box is found upstream from nucleotide 1 . A GC box (Kadonaga et al. 1986) in the 5 '-genomic sequence, the consensual polyadenylation signal AATAAA (Proudfoot 1984), and a cluster of four reported polyadenylation signals downstream, AATTAA and ATTAAA (Birnstiel et al. 1985), are underlined. Exons 3 and 9 are alternative; they are present in cDNA Al6 and absent in the other three cDNAs. Exons $6 \mathrm{a}$ and $6 \mathrm{~b}$ are mutually exclusive because the open reading frame is incompatible with their simultaneous presence. $\mathrm{cDNAs}$ E2, L9, and A6 contain exon $6 \mathrm{a}$, while cDNA A16 contains exon 6b. Exon 10 includes a consensual polyadenylation signal at position 1184-1189 (Proudfoot 1984). E2 and A16 cDNAs are polyadenylated 27 nucleotides downstream of this signal. L9 and A6 cDNAs extend 170 nucleotides farther downstream. This additional sequence represents an extended version of exon 10. Consequently, a second polyadenylation site, generating longer mRNAs, should exist. Although the precise location of this second site is not yet known, the accumulation of other polyadenylation signals at positions 1365-1376, together with the actual size of the mRNAs observed in Northern blots (see Fig. 5), suggests that this site can be included in exon 10 and probably not far from the 3' end of cDNAs L9 and A6.

ertheless, in this case the region of maximum homology persists in the actin/TnC-binding domain (see Fig. 6; Van Eyk and Hodges 1988). The criteria used by Buschmeier et al. (1987) to group the calmodulin-binding domains of different proteins are satisfied by this Drosophila TnI.

The domain encoded in exon 3 has no homology with any $\mathrm{TnI}$ reported so far. Albeit, it has a remarkable similarity with two proline- and alanine-enriched domains found in some Drosophila Tm isoforms (Karlik et al. 1984). These Tm domains seem to be expressed specifically in the flight muscles (Karlik and Fyrberg 1986). Accordingly, we find that exon 3 is adult specific.

Exon 10 gives a carboxyl terminus containing a tryptophan residue that is conserved at the same position in all vertebrate $\mathrm{TnI}$ isoforms known so far. The alternative carboxyl terminus encoded in exon 9 lacks this tryptophan residue.

\section{Discussion}

We have shown that the HL I complementation group of the HL region in the $S h C$ is defined by viable mutations $(h d p)$, as well as recessive [1(1)1614], semidominant [1(1)8384], and dominant [1(1)9916] lethal mutations. Two of these mutations have been found to map physically within a transcription unit that encodes different isoforms of TnI. The mutant phenotypes show abnormal development of preblastoderm embryo, muscular, and neural tissues. In addition, l(1)1614 has been shown to alter action potential profiles (Ferrús et al. 1990).

To our knowledge, this is the first report of mutations in a TnI gene that cause neural phenotypes. The existence of mutant phenotypes in the nervous system and the failure to detect TnI RNA expression in this tissue might be of some concern. However, because RNA in situ hybridization tests are far less sensitive than radio- 


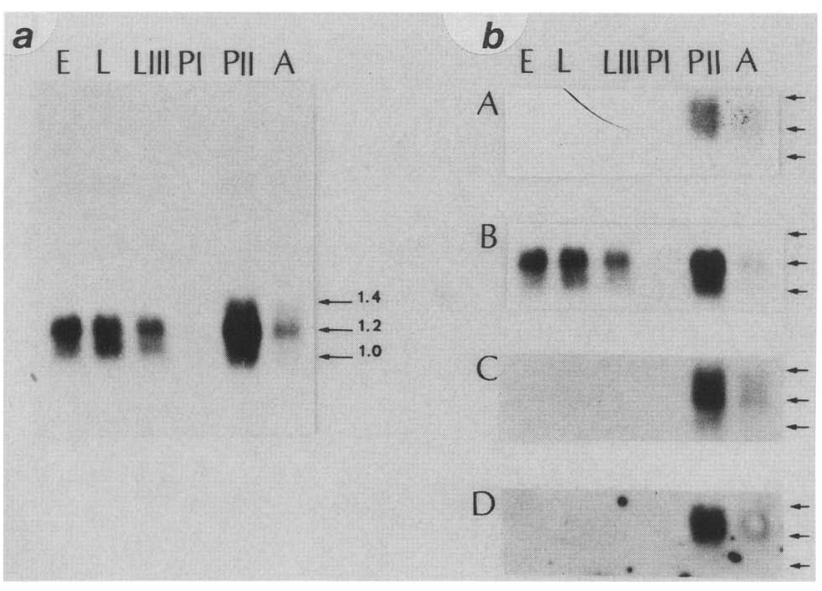

Figure 5. Developmental mRNA blots. Poly $(A)^{+}$RNAs from different developmental stages were hybridized with the following probes: $(a)$ complete E2 cDNA; $(b)$ exon $3(A)$, exon 6a $(B)$; exon $6 \mathrm{~b}(C)$; exon $9(D)$. The extension of probes $A-D$ is shown in Fig. 3 (see also Materials and methods). Arrows point to the three different mRNA bands $(1.4,1.2$, and $1.0 \mathrm{~kb})$ in all cases. (E) Embryos; (L) first- and second-instar larvae; (LIII) third-instar larvae; (PI) P7-P9 stages; (PII) P12-P14 stages (Bainbridge and Bownes 1981); (A) adults. All hybridizations were carried out on the same blot except in $b(D)$.

immunoassays with protein isoform-specific probes, one should analyze these data before determining the direct presence of $\mathrm{TnI}$ in the nervous system. The possibility that the neural phenotypes are caused by a second site mutation or a polar effect on adjacent transcriptional units appears unlikely because it would be necessary for three mutations, 1(1)1614, 1(1)8384, and 1(1)9916, to have the same effect. We maintain that the entire $S h C$ is composed of functionally related transcription units. It is important to realize that the analysis of complementation is a functional test and the complementation groups are not necessarily identical to transcription units. The fact that all of the mutations described here form a single complementation group demonstrates that the corresponding products are functionally related to
TnI independently of the actual physical location of these mutations.

\section{TnI during development}

The extensive homology of HL I structural products with the reported sequence of invertebrate TnI makes it very plausible that the described mutant phenotypes are causally related to Drosophila TnI. The aborted embryogenesis observed in the dominant lethal 1(1)9916 mutation (Fig. 1E), an inversion that breaks within the transcription unit, could possibly be related to a complete depletion of TnI. Thus, it appears that $\mathrm{TnI}$ is required for early embryogenesis, perhaps as a constituent of the cytoskeleton. In addition, TnI is needed for maintenance of muscle structure. Notably, the fibrillar muscles of the thorax (DLM and DVM) are the most sensitive to TnI defects. The 1(1)1614 causes a dominant and specific effect on the structure of the tubular muscle TDT. However, other tubular muscles (i.e., proboscis and abdomen) do not appear to be affected in the 1(1)1614/ + or $h d p$ mutants. This phenomenon of muscle specificity has been described for other Drosophila genes, such as stripe, whose product is required for muscle development (De la Pompa et al. 1989). Because alleles of the same complementation group cause specific muscle phenotypes, it is likely that different muscles would require specific $\mathrm{TnI}$ isoforms. Although the molecular consequences of the $h d p$ mutations on HL I transcription are still unknown, the developmental expression of exon 3 (Fig. 5) coincides with the temperature-sensitive period of the temperature-sensitive allele $h d p^{101}$ (Homyk and Emerson 1988).

In addition to these requirements of TnI in muscle development, it seems that TnI function(s) is also required in neurons. The aberrant neurogenesis found in 1(1)8384 and 1(1)9916 supports this conclusion. In addition, TnI may be required by mature neurons, as suggested by the abnormal action potentials and shaking activity of 1(1)1614/ + adults (Fig. 2). The structural nature of TnI indicates that it might be involved in the cytoskeletal apparatus. It is plausible that in this case, a TnI isoform

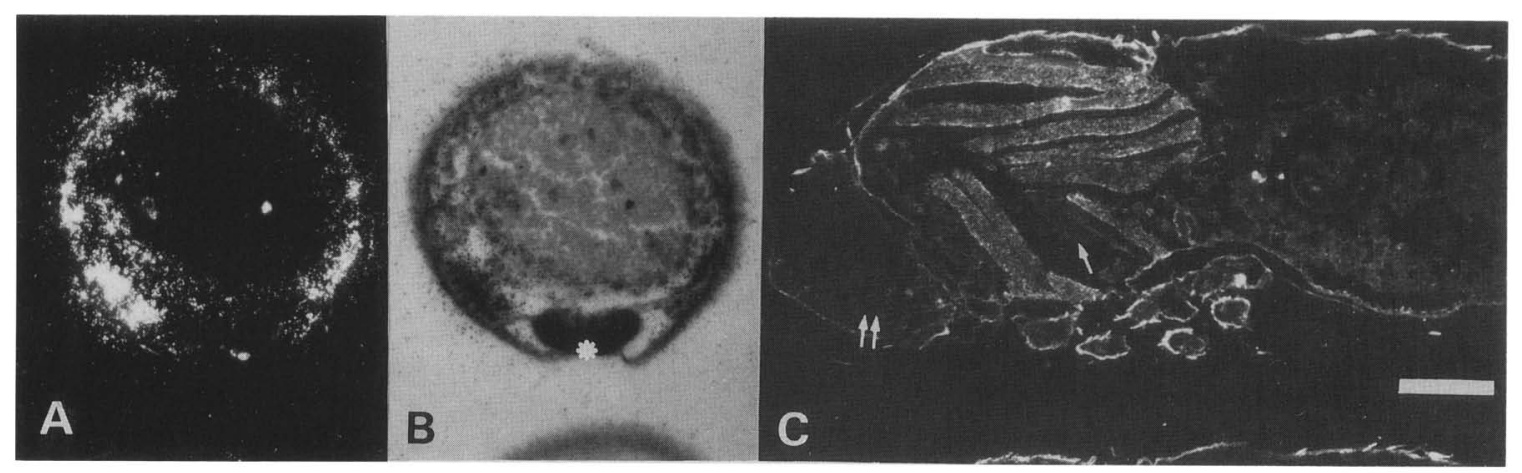

Figure 6. In situ expression of HL I products. (A) Frontal section of a 12-hr embryo hybridized with an HL I riboprobe (cDNA E2) common to all isoforms known to date. The signal is detected in the mesoderm. (B) Bright-field view of $A$ (asterisk marks the CNS). (C) Sagittal section of a wild-type adult hybridized with an exon 3-specific riboprobe. The signal is detected in DLM and DVM fibrillar muscles but not in TDT (single arrow) nor in proboscis (double arrows) tubular muscles. Bar, $70 \mu \mathrm{m}$ in $A$ and $B$ and $500 \mu \mathrm{m}$ in $\mathrm{C}$. 


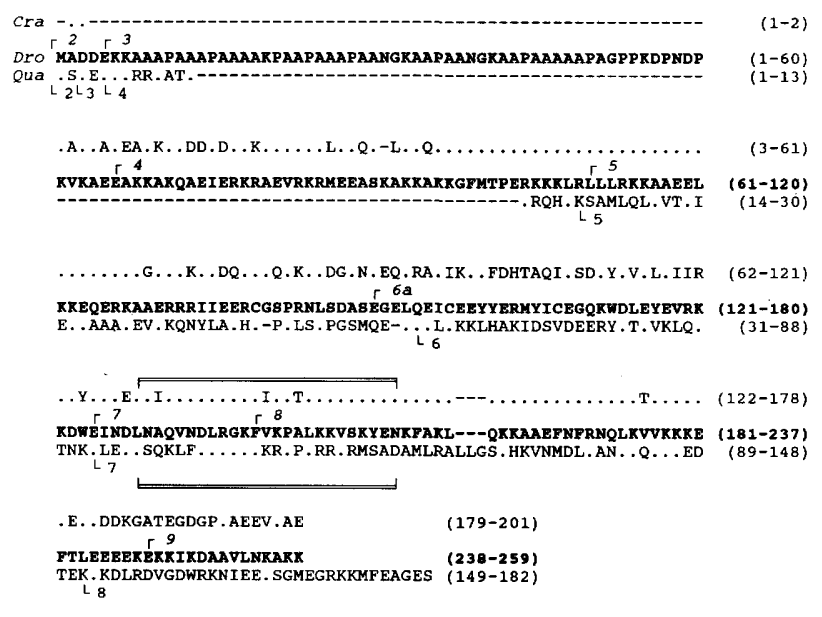

Figure 7. Alignment of the amino acid sequences of different TnIs. (Top) Crayfish (Kobayashi et al. 1989); (middle) Drosophila (p36b9); (bottom) quail fast skeletal muscle (Baldwin et al. 1985). Dots in the top and bottom rows mean identity with the sequence shown in the middle. Sequences of Drosophila and crayfish are divided accordingly with the coding exons. The box encloses the actin/TnC-binding domains and includes the phosphorylation site serine (S) 118 in the quail sequence. Homologous S is found in Drosophila (residue 208) and in crayfish (residue 149), corresponding to potential phosphorylation sites for the CAMP/cGMP-dependent protein kinase (Kobayashi et al. 1989). Exon 2 in Drosophila encodes the first 5 amino acids of the protein. Only identities or conservative changes are observed in the corresponding region of quail, although it is split into two exons (2 and 3). At the nucleotide level, 13 of the 15 derived amino acids are identical (not shown). Exon 3 of Drosophila (only present in adult mRNAs) has no counterpart in vertebrates or crayfish TnI reported so far. Exon 4 has its counterpart in crayfish, but no similar sequences are observed in vertebrates. Interestingly, exon 4 of quail has some similarity with the sequence of Drosophila but is excised in two parts. The first half can be paired with the amino border of exon 3 of Drosophila, whereas the second half can be paired with the carboxyl border of exon 4. A putative secondary TnC-binding domain (Perry 1979) is supposed to be encoded in exon 4 of quail. This domain is not found in Drosophila. Both exons 5 and 6 (either forms a or b) have similar lengths in both species, although sequence similarity decreases. Quail exon 7 encodes the actin/ TnC-binding domain (amino acids 98-120). This binding domain is split into exons 7 and 8 of Drosophila. Strikingly, the splice site coincides with parts A and B in which some investigators divide the calmodulin-binding domains (Buschmeier et al. 1987). It seems that the sequences of primary and secondary TnC-binding domains, as defined in vertebrate TnI (Perry 1979), are maintained in Drosophila, although the corresponding DNA appears to be interrupted by introns. (A) Alanine; (G) glutamate; $(\mathrm{K})$ lysine; $(\mathrm{L})$ leucine; $(\mathrm{P})$ proline; (W) tryptophan.

could be functionally coupled to the $\mathrm{K}^{+}$channels encoded in the V region of ShC. Precedents of cytoskeletal proteins coupled to ionic channels are known for the $\mathrm{Na}^{+}$channel and the acetylcholine receptor (Edelstein et al. 1988; Flucher and Daniels 1989). Although the confirmation of these hypothetical interactions would require a long series of physiological and biochemical tests, it is evident that the molecules involved should have structural features to sustain physical interactions.

\section{A possible structural base for multimeric interactions of $T n I$}

The functional relationships among the $S h C$ proteins suggested by the genetic analysis of the region (Ferrús et al. 1990) would require the formation of heteromultimers. In this context, it should be noted that the deduced amino acid sequence of TnI shows a modified version of the leucine zipper motif. TnI has a periodic repetition of leucine residues at every seventh position between amino acids 167 and 195 (Fig. 7). The periodicity is only broken at position 181 where a lysine substitutes the leucine. The leucine and lysine residues are always flanked by charged amino acids, giving the motif ${ }^{*}-\mathrm{L}^{-}{ }^{*}-X-X-X-X{ }^{-*}-\mathrm{L}^{*}-X-X-X-X-^{*}{ }^{-}{ }^{*}-{ }^{*}-X-X-X-X-{ }^{*}-\mathrm{L}^{-}-X-X-$ $X-X^{*}-\mathrm{L}^{*}$, where ${ }^{*}$ represents a charged residue, $\mathrm{L}$ represents leucine, and $X$ represents any amino acid. Strikingly, a similar motif is found in myosin, that is, residues 1026-1057 of the myosin heavy chain of embryonic rat skeletal muscle (Strehler et al. 1986). Furthermore, in this region of myosin, the leucine periodicity is broken at the homologous position of TnI by a glutamate residue. This motif is very similar to the periodic array of leucine described in other proteins and proposed to participate in the formation of leucine zippers (Landschulz et al. 1988), a structure that stabilizes an $\alpha$-helix conformation via interaction between two different chains. Interestingly, the $\mathrm{K}^{+}$channels encoded in the $\mathrm{V}$ region of the $S h C$ exhibit another leucine zipper motif between domains S4 and S5 (see Fig. 2 in Pongs et al. 1988).

The intended role of these leucine zippers might be extended to cases in which the leucine "spoke" is interrupted by a charged residue, as long as an opposite charge is found in the corresponding site of the leucine spoke of the interacting chain. Indeed, this modified version of the leucine spokes is found also in $\mathrm{TnT}$ and myosin heavy-chain tail (deduced from Breitbart and Nadal-Ginard 1986 and Strehler et al. 1986). This class of leucine-mediated interactions perhaps represents a general mechanism of interaction in protein multimers.

\section{Significance of haplolethality}

Haplolethality is an unusual phenomenon in Drosophila. Aside from the HL region of ShC, another HL region is located at chromosome band $88 \mathrm{~F}$ (Lindsley et al. 1972). The HL region at $88 \mathrm{~F}$ encodes several forms of Tm and a form of actin (Karlik et al. 1984). The similarity between the alanine- and proline-rich exon 3 of TnI and the alanine- and proline-rich domain of some Tm isoforms encoded in $88 \mathrm{~F}$ (Karlik and Fyrberg 1986) is noticeable. In addition, these isoforms of $\mathrm{TnI}$ and $\mathrm{Tm}$ are adult specific. These two HL regions have been claimed to be functionally related (Homyk and Emerson 1988). A thorough genetic and mutational analysis of $88 \mathrm{~F}$ should provide the required data to establish the potential relation- 
ships between the two regions. The mutational analysis of the HL region of ShC has yielded several dominant lethal mutations (Ferrús et al. 1990). However, it is not yet known whether all of them map into the same or different transcription units.

We find it unlikely that a $50 \%$ reduction in the amount of TnI would cause dominant lethality to Drosophila. The HL I products could perform independent regulatory functions on the expression or activity of other proteins. The shared homology with calmodulinbinding centers could be the basis for these regulatory functions (Rasmussen and Means 1989). Along this line, the functional organization of the entire $S h C$ could be understood.

\section{Materials and methods}

Fly strains, developmental studies, and genotype identification

Normal flies were from the Canton-S stock. Lethal mutations were induced on a $f^{5}$ os-marked chromosome as described in Ferrús et al. (1990). Descriptions of muscle phenotypes were based on $10-\mu \mathrm{m}$ sections of paraffin-embedded flies stained with Giemsa, toluidine blue, or Milligan's trichrome methods (Humason 1972). A minimum of five serially sectioned flies were studied per each genotype. The following genotypes were analyzed as sectioned material: female $1 / 1) 1614 /+$; $D p(1 ; 3) / C 153 /+$, female $1(1) 1614 /+$, female $1(1) 1614 / 1(1) 9916$; $D p(1 ; 3) / C 153 /+$, female $h d p^{3 / 1}(1) 8384 ; D p(1 ; 3) / C 153 /+$, male $h d p^{2}$, male $h d p^{3}$, female $h d p^{2} / 1(1) 1614$, female $h d p^{3} / 1(1) 1614$, female $h d p^{3} / 1(1) 8384$. These genotypes are listed in increasing order of muscle phenotype severity. Observations of the CNS were carried out in dissected embryos, stained with $\mathrm{mAb}$ $22 \mathrm{C} 10$. In vivo studies of early development were as in Wieschaus and Nüsslein-Volhard (1986), and CNS whole mounts were as in Canal and Ferrús (1986).

\section{cDNA libraries, DNA labeling, and sequencing}

Embryo (3- to 12-hr) and third-instar larvae cDNA libraries were a gift from L.M. Kauvar (see Poole et al. 1985). The adult cDNA library was a gift from S. Orgad and Y. Dudai (The Weizmann Institute, Israel). Restriction fragments of recombinant phages were subcloned into pUC19 or Bluescript (Stratagene) by standard cloning techniques (Sambrook et al. 1989).

DNA probes for library screening or hybridization to Northern and Southern blots were labeled with $\left[\alpha^{-32} \mathrm{P}\right] \mathrm{dATP}$ ( $3000 \mathrm{Ci} / \mathrm{mmole}$ ) by the random primer method (Feinberg and Vogelstein 1983). Nucleotide sequences were determined by the dideoxy chain-terminating method (Sanger et al. 1977) using the Sequenase sequencing kit (U.S. Biochemicals). In some cases, specific oligonucleotides of the HL I transcription unit were synthesized and used as primers for sequencing. Both strands were sequenced in the case of cDNA inserts. For sequence homology comparison, we used the FASTA program (Pearson and Lipman 1988).

\section{Developmental Northern blots and in situ hybridization}

Whole RNA was extracted from different developmental stages using the guanidine thiocyanate-phenol method (Chomczynski and Sacchi 1987). Poly(A)+ RNA was selected using oligo(dT)-cellulose (Boehringer) under conditions suggested by the supplier. RNA was electrophoresed onto nylon membranes (Amersham) and hybridized in 50\% formamide, $6 \times$ SSC, $10 \times$
Denhardt's solution, and $100 \mu \mathrm{g} / \mathrm{ml}$ single-stranded sperm DNA. Probes were labeled to a sp. act. of $5 \times 10^{8} \mathrm{cpm} / \mu \mathrm{g}$ and were used at $2 \times 10^{6} \mathrm{cpm} / \mathrm{ml}$. Final wash was in $0.2 \times \mathrm{SSC}$, $0.1 \%$ SDS, at $65^{\circ} \mathrm{C}$. Exposure time ranged between several hours to 3 days. Probes were removed by boiling water, and effectiveness was checked by exposing the filter for 2 days.

For exon-specific hybridization the following probes were prepared. For exons 2 and $6 \mathrm{~b}$ we used the genomic fragments A and D, respectively, in Figure 3. They were labeled by the random primer method described above. For exon 9 we used an antisense synthetic oligodeoxinucleotide (5'-ATTTAGCACAGCGGCATCTT-3'). It was labeled with [ $\gamma^{32}$ P]ATP $(3000$ $\mathrm{Ci} / \mathrm{mmole}$ ) and the $\mathrm{T} 4$ polynucleotide kinase as described in Sambrook et al. (1989). Hybridization was carried out overnight at $42^{\circ} \mathrm{C}$ in $35 \%$ formamide, $50 \mathrm{mM}$ Tris- $\mathrm{HCl}(\mathrm{pH} 7.5), 1 \mathrm{M} \mathrm{NaCl}$, $50 \mu \mathrm{g} / \mathrm{ml}$ single-stranded sperm DNA; $150 \mu \mathrm{g} / \mathrm{ml}$ yeast tRNA, and $5 \times$ Denhardt's solution. Washes were in $3 \times \mathrm{SSC} ; 0.1 \%$ SDS, at room temperature. For exon $6 a$ we first amplified the whole exon by the PCR, using standard protocols (Sambrook et al. 1989) and then labeled the fragment as described by random primer.

In situ hybridizations were carried out in $10-\mu \mathrm{m}$ sections from paraffin-embedded embryos or adults using ${ }^{35} \mathrm{~S}$-labeled antisense RNA probes following the procedure described in Pongs et al. (1988).

\section{Acknowledgments}

We are grateful to our colleague F. Tejedor for constructive criticisms and M.A. Arévalo for oligonucleotide synthesis. J.A.B. is recipient of a fellowship from Fondation pour l'Etude du Systeme Nerveux Central et Périphérique, Geneva, Switzerland). This work was supported by grants from CAICYT (A.F.), VW Stiftung and EEC (A.F. and O.P), and DFG (O.P.).

The publication costs of this article were defrayed in part by payment of page charges. This article must therefore be hereby marked "advertisement" in accordance with 18 USC section 1734 solely to indicate this fact.

\section{References}

Bainbridge, S.P. and M. Bownes. 1981. Staging the metamorphosis of Drosophila melanogaster. I. Embryol. Exp. Morphol. 66: 57-80.

Baldwin, A.S. Jr., E.L.W. Kittler, and C.P. Emerson Jr. 1985. Structure, evolution, and regulation of a fast skeletal muscle troponin I gene. Proc. Natl. Acad. Sci. 82: 8080-8084.

Baumann, A., I. Krah-Jentgens, R. Müller, F. Müller-Holtkamp, R. Seidel, N. Kecskemethy, J. Casal, A. Ferrús, and O. Pongs. 1987. Molecular organization of the maternal effect region of the Shaker complex of Drosophila: Characterization of an $\mathrm{I}_{\mathrm{A}}$ channel transcript with homology to vertebrate $\mathrm{Na}^{+}$ channel. EMBO I. 6: 3419-3429.

Birnstiel, M.L., M. Busslinger, and K. Strub. 1985. Transcription termination and $3^{\prime}$ processing: The end is in site! Cell 41: 349-359.

Breitbart, R.E. and B. Nadal-Ginard. 1986. Complete nucleotide sequence of the fast skeletal troponin $\mathrm{T}$ gene. Alternatively spliced exons exhibit unusual interspecies divergence. $/$. Mol. Biol. 188: 313-324.

Buschmeier, B., H.E. Meyer, and G.W. Mayr. 1987. Characterization of the calmodulin-binding sites of muscle phosphofructokinase and comparison with known calmodulinbinding domains. J. Biol. Chem. 262: 9454-9462.

Canal, I. and A. Ferrús. 1986. The pattern of early neuronal dif- 
ferentiation in D. melanogaster. J. Neurogenet. 3: 293-319. Chomczynski, P. and N. Sacchi. 1987. Single-step method of RNA isolation by acid guanidinium thiocyanate-phenolchloroform extraction. Anal. Biochem. 162: 156-159.

Deak, I.I., P.R. Bellamy, M. Bienz, Y. Dubuis, E. Fenner, M. Gollin, A. Rähmi, Th. Ramp, Ch.A. Reinhardt, and B. Cotton. 1982. Mutations affecting the indirect flight muscles of Drosophila melanogaster. J. Embryol. Exp. Morphol. 69: 61-81.

de la Pompa, J.L., J.R. García, and A. Ferrús. 1989. Genetic analysis of muscle development in Drosophila melanogaster. Dev. Biol. 131: 439-454.

Edelstein, N.G., W.A. Catterall, and R.T. Moon. 1988. Identification of a 33-kilodalton cytoskeletal protein with high affinity for the sodium channel. Biochemistry 27: 1818-1822.

Feinberg, A.P. and B. Vogelstein. 1983. A technique for radiolabelling DNA restriction endonuclease fragments to high specific activity. Anal. Biochem. 132: 6-13.

Ferrús, A., S. Llamazares, J.L. de la Pompa, M.A. Tanouye, and O. Pongs. 1990. Genetic analysis of the Shaker gene complex of Drosophila melanogaster. Genetics 125: 383-398.

Flucher, B.E. and M.P. Daniels. 1989. Distribution of $\mathrm{Na}^{+}$ channels and ankyrin in neuromuscular junctions is complementary to that of acetylcholine receptors and the $43 \mathrm{kd}$ protein. Neuron 3: 163-175.

Fujita, S.C., S.L. Zipursky, S. Benzer, A. Ferrús, and S.L. Shotwell. 1982. Monoclonal antibodies against the Drosophila nervous system. Proc. Natl. Acad. Sci. 79: 7929-7933.

Homyk, Th. and Ch.P. Emerson. 1988. Functional interactions between unlinked muscle genes within haploinsufficient regions of the Drosophila genome. Genetics 119: 105-121.

Hotta, Y. and S. Benzer. 1972. Mapping of behaviour in Drosophila mosaics. Nature 240: 527-535.

Humason, G.L. 1972. Animal tissue techniques. W.H. Freeman, San Francisco.

Iverson, L.E., M.A. Tanouye, H.A. Lester, N. Davidson, and B. Rudy. 1988. Potassium channels from Shaker RNA expressed in Xenopus oocytes. Proc. Natl. Acad. Sci. 85: $5723-5727$.

Kadonaga, J.T., K.A. Jones, and R. Tjian. 1986. Promoter-specific activation of RNA polymerase II transcription by $\mathrm{Spl}$. Trends Biochem. Sci. 11: 20-23.

Karlik, Ch.C. and E.A. Fyrberg. 1986. Two Drosophila melanogaster tropomyosin genes: Structural and functional aspects. Mol. Cell. Biol. 6: 1965-1973.

Karlik, Ch.C., J.W. Mahaffey, M.D. Coutu, and E.A. Fyrberg. 1984. Organization of contractile protein genes within the $88 \mathrm{~F}$ subdivision of the $D$. melanogaster third chromosome. Cell 37: 469-481.

Kobayashi, T., T. Tagaki, K. Konishi, and J.A. Cox. 1989. Amino acid sequence of crayfish troponin I. J. Biol. Chem. 264: $1551-1557$.

Kozak, M. 1984. Compilation and analysis of sequences upstream from the translational start site in eukaryotic mRNAs. Nucleic Acids Res. 12: 857-872.

Landschulz, W.H., P.F. Johnson, and S.L. McKnight. 1988. The leucine zipper: A hypothetical structure common to a new class of DNA binding proteins. Science 240: 1759-1764.

Lindsley, D.L., L. Sandler, B.S. Baker, A.T.C. Carpenter, R.E. Denell, J.C. Hall, P.A. Jacobs, G.L.G. Miklos, B.K. Davis, R.C. Gethmann, R.W. Hardy, A. Hessler, S.M. Miller, H. Nozawa, D.M. Parry, and G. Somero. 1972. Segmental aneuploidy and the genetic gross structure of the Drosophila genome. Genetics 71: 157-184.

Padgett, R.A., P.J. Grabowski, M.M. Konarska, S. Seiler, and P.A. Sharp. 1986. Splicing of messenger RNA precursors.
Annu. Rev. Biochem. 55: 1119-1150.

Pearson, W.R. and D.J. Lipman. 1988. Improved tools for biological sequence comparison. Proc. Natl. Acad. Sci. 85: 24442448.

Perry, S.V. 1979. The regulation of contractile activity in muscle. Biochem. Soc. Trans 7: 593-617.

Pongs, O., N. Kecskemethy, R. Müller, I. Krah-Jentgens, A. Baumann, H.H. Kiltz, I. Canal, S. Llamazares, and A. Ferrús. 1988. Shaker encodes a family of putative potassium channel proteins in the nervous system of Drosophila. EMBO I. 7: 1087-1096.

Poole, S.J., L.M. Kauvar, B. Drees, and T. Kornberg. 1985. The engrailed locus of Drosophila: Structural analysis of an embryonic transcript. Cell 40: $37-43$.

Proudfoot, N. 1984. The end of message and beyond. Nature 307: $412-413$.

Rasmussen, C.D. and A.R. Means. 1989. Calmodulin, cell growth and gene expression. Trends Neurosci. 12: 433-438.

Sambrook, J., E.F. Fritsch, and T. Maniatis. 1989. Molecular cloning: A laboratory manual, Cold Spring Harbor Laboratory Press, Cold Spring Harbor, New York.

Sanger, F., S. Nicklen, and A.R. Coulson. 1977. DNA sequencing with chain-terminating inhibitors. Proc. Natl. Acad. Sci. 74: 5463-5467.

Schwartz, Th.L., B.L. Tempel, D.M. Papazian, Y.N. Jan, and L.Y. Jan. 1988. Multiple potassium channels components are produced by alternative splicing at the Shaker locus in Drosophila. Nature 331: 137-142.

Strehler, E.E., M.-A. Strehler-Page, J.-C. Perriard, M. Periasamy, and B. Nadal-Ginard. 1986. Complete nucleotide and encoded amino acid sequence of a mammalian myosin heavy chain gene. Evidence against intron-dependent evoluation of the rod. J. Mol. Biol. 190: 291-317.

Tanouye, M.A. and A. Ferrús. 1985. Action potentials in normal and Shaker mutant Drosophila. I. Neurogenet. 2: 253-271.

Tanouye, M.A., A. Ferrús, and S.C. Fujita. 1981. Abnormal action potentials associated with the Shaker complex locus of Drosophila. Proc. Natl. Acad. Sci. 78: 6548-6552.

Van Eyk, J.E. and R.S. Hodges. 1988. The biological importance of each amino acid residue of the troponin I inhibitory sequence $104-115$ in the interaction with troponin $\mathrm{C}$ and tropomyosin-actin. I. Biol. Chem. 263: 1726-1732.

Wieschaus, E. and Ch. Nüsslein-Volhard. 1986. Looking at embryos. In Drosophila: A practical approach pp. 198-227. (ed. D.B. Roberts). IRL Press, Oxford. 


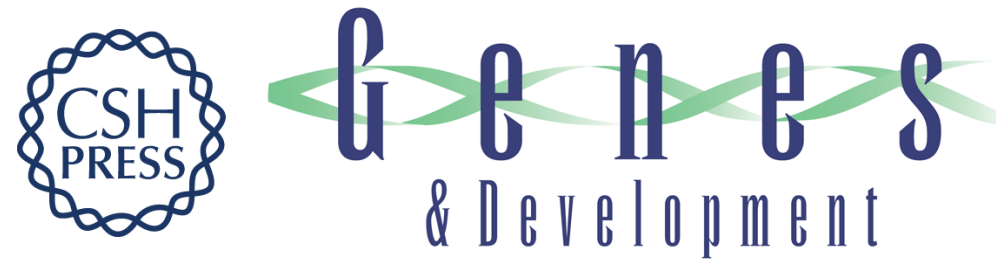

\section{Troponin I is encoded in the haplolethal region of the Shaker gene complex of Drosophila.}

J A Barbas, J Galceran, I Krah-Jentgens, et al.

Genes Dev. 1991, 5:

Access the most recent version at doi:10.1101/gad.5.1.132

References This article cites 38 articles, 15 of which can be accessed free at:

http://genesdev.cshlp.org/content/5/1/132.full.html\#ref-list-1

License

Email Alerting Receive free email alerts when new articles cite this article - sign up in the box at the top Service right corner of the article or click here.

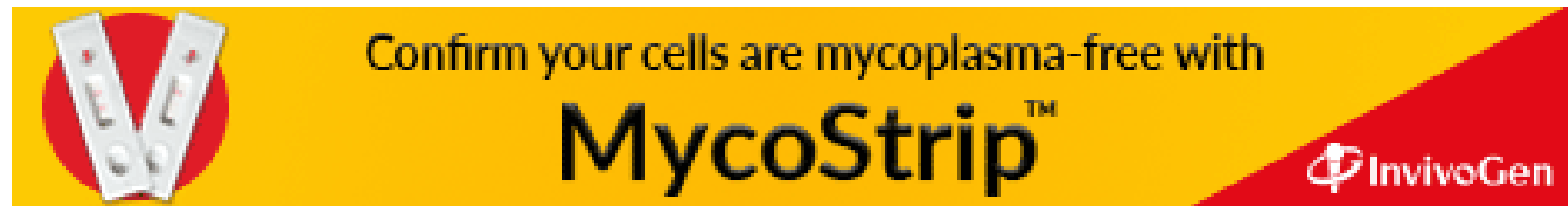

